\title{
Endovascular Management of Stroke Patients with Large Vessel Occlusion and Minor Stroke Symptoms
}

Saeed A. Alqahtani ${ }^{1}$, Andrew B. Stemer ${ }^{2}$, Michael F. McCullough ${ }^{3}$, Randy S. Bell ${ }^{4}$, Jeffrey Mai ${ }^{1}$, Ai-Hsi Liu ${ }^{3}$, Rocco A. Armonda ${ }^{1}$

1. Neurosurgery, Medstar Georgetown University Hospital 2. Neurology, Medstar Georgetown University Hospital 3. Neurointerventional Radiology, Medstar Washington Hospital Center 4. Department of Neurosurgery, Walter Reed Army Medical Center, Washington D.C.

$\square$ Corresponding author: Saeed A. Alqahtani, saeedkanee@gmail.com Disclosures can be found in Additional Information at the end of the article

\section{Abstract}

Endovascular mechanical thrombectomy for stroke patients with large vessel occlusion (LVO) in the anterior circulation has become the standard of care based on several major randomized clinical trials. The successful result reported by these trials constitutes what may be the largest achievement in the history of neurological sciences. However, most of these mechanical thrombectomy trials (except for the multicenter randomized clinical trial of endovascular treatment for acute ischemic stroke in the Netherlands, i.e., MR CLEAN and Extending the Time for Thrombolysis in Emergency Neurological Deficits-Intra-Arterial, i.e., EXTEND-IA) excluded stroke patients with minor to mild stroke symptoms with National Institutes of Health Stroke Scale (NIHSS) scores of six to eight or lower. The median NIHSS score for patients who underwent acute endovascular thrombectomy was approximately 15 to 17 in all trials. To date, the evidence is lacking to support the mechanical thrombectomy in patients with acute stroke and LVO with minor to mild severity on NIHSS score. The purpose of this review was to assess the current data, safety and clinical outcomes in stroke patients with minor to mild symptoms who were treated with endovascular thrombectomy.

Categories: Neurology, Neurosurgery

Keywords: ischemic stroke, large vessel occlusion, endovascular intervention, mechanical thrombectomy, tpa, outcomes

\section{Introduction And Background}

Received 06/08/2017 Review began 06/09/2017 Review ended 06/12/2017 Published 06/15/2017

\section{C) Copyright 2017}

Alqahtani et al. This is an open access article distributed under the terms of the Creative Commons Attribution License CC-BY 3.0., which permits unrestricted use, distribution, and reproduction in any medium, provided the original author and source are credited.
An untreated large vessel occlusion (LVO) in acute ischemic stroke is associated with poor clinical outcomes. Approximately $80 \%$ of patients with an untreated LVO will die within 90 days or become functionally disabled [1]. Treatment with intravenous tissue plasminogen activator (tPA) alone in patients with LVO is associated with a low recanalization rate [2]. For this reason, mechanical thrombectomy along with intravenous tPA has become the standard treatment of acute ischemic stroke patients with LVO and moderate to severe stroke symptoms [3-4]. The recent major clinical trials were mainly focused on patients with moderate to severe stroke symptoms except for the multicenter randomized clinical trial of endovascular treatment for acute ischemic stroke in the Netherlands (MR CLEAN) and Extending the Time for Thrombolysis in Emergency Neurological Deficits-Intra-Arterial (EXTEND-IA) trials [5-9].

There are a significant number of patients with acute ischemic stroke and LVO who can present with mild neurological symptoms. The complex interplay between LVO, blood pressure,

How to cite this article

Alqahtani S, Stemer A B, Mccullough M F, et al. (June 15, 2017) Endovascular Management of Stroke

Patients with Large Vessel Occlusion and Minor Stroke Symptoms. Cureus 9(6): e1355. DOI

10.7759/cureus.1355 
collateral status, and clinical symptomatology can result in some patients with LVO presenting with low NIHSS scores. In a large prospective study, higher NIHSS scores had a merely $48 \%$ sensitivity for predicting LVO [10]. Most patients present with low NIHSS scores and minimal neurological deficits should not exclude angiographic evaluation in patients with acute stroke symptoms [10-11]. The direct visualization of LVO is extremely important and the treatment modality should include mechanical thrombectomy in appropriate clinical scenarios because the potential recanalization rate with intravenous tPA alone is very low and the chance for neurological recovery is unfavorable.

Based on recent major mechanical thrombectomy trials, the American Heart Association guidelines provided level 1a evidence for mechanical thrombectomy for patients with NIHSS scores of six or more [12]. However, in a large nationwide study, many patients did not receive tPA or early angiographic imaging because of their low NIHSS scores or because, their initial stroke symptoms improved, yet they had poor neurological outcomes [13]. To date, no randomized clinical trials focus on patients with acute ischemic stroke with LVO and low NIHSS scores. The aim of this review is to assess current clinical outcomes and the safety of endovascular mechanical thrombectomies in these patients.

\section{Review}

An acute ischemic stroke with LVO could present with minor stroke symptoms that may result in severe disability or even death if left untreated. Rapidly improving or minor initial stroke symptoms are the most common cause for excluding patients from acute recanalization therapy [14]. One large published study reported $31.2 \%$ of patients with acute ischemic stroke did not receive intravenous tPA because of mild or rapidly improving stroke symptoms. Among those with mild strokes or improving symptoms who did not receive therapy, $28.3 \%$ were not discharged to home and $28.5 \%$ were not able to ambulate and experienced a poor clinical outcome at 90 days [14-18].

Maas, et al. reported that the National Institutes of Health Stroke Scale (NIHSS) scores with > 10 have approximately $81 \%$ positive predictive value for large proximal occlusion, but only $48 \%$ sensitivity with most of the patients with LVO presenting with lower NIHSS scores were found ( $55 \%$ of patients with NIHSS scores $\leqslant 10$ ). They concluded that no such NIHSS score threshold could be applied to select a subgroup of stroke patients for acute angiographic imaging without failing to capture most patients with LVO. Failure to visualize the proximal vessel occlusion in acute stroke patients with minor stroke symptoms could have a devastating neurological outcome knowing that the intravenous tPA alone has almost no significant recanalization effect in large proximal occlusion [10]. In these cases, > 60\% of the patients will die within 90 days or become functionally disabled [19].

Kim, et al.'s retrospective study of 378 patients with acute ischemic stroke and mild symptoms reported that early neurological deterioration occurred in 55 patients (14.6\%) and symptomatic large arterial occlusion was independently associated with neurological deterioration in patients with mild initial stroke presentation [20].

Nedeltchev, et al. studied 162 patients presenting within six hours of acute ischemic stroke onset who did not receive acute thrombolysis therapy because of mild or rapidly improving symptoms. The authors reported 38 patients (23.5\%) experienced poor outcomes and $1.3 \%$ of patients had died. The presence of proximal LVO drives the chances of poor neurological outcomes to 7.13-fold [18].

Mokin, et al. noted the variability seen in the neurological outcomes of 204 acute ischemic stroke patients with LVO who were admitted with low baseline NIHSS scores (range zero to 
seven) and excluded from acute intravenous thrombolysis and endovascular intervention. The study reported an increase in disability at the time of discharge for patients with NIHSS scores of zero to two [21].

There is also some evidence to suggest that stroke location may present with falsely mild symptoms (e.g., right-sided infarcts) and poor outcomes without thrombolytic or endovascular treatment. In a study by Cronin, et al, of 66 patients excluded from acute thrombolysis therapy because of rapidly improving or mild symptoms, $16.7 \%$ had poor outcomes. Six patients with neurologic deficits had right hemispheric infarcts and one patient also had a cerebellar stroke $[22]$.

The rapidly improving or minor initial deficit is the most common etiology for excluding acute ischemic stroke patients with LVO from thrombolytic therapy. However, the notion of the complex role of leptomeningeal collateral status and good clinical outcomes may affect the decision for mechanical thrombectomy. There is some evidence to support this notion. However, the good collateral status alone is not always optimal and sufficient. As always, recanalization remains critical [23-26].

Miteff, et al. studied 92 acute ischemic stroke patients with LVO and found good collaterals in approximately $55 \%$ of patients. The study demonstrated that patients with good collaterals who received an endovascular thrombectomy achieved favorable outcomes. However, in patients with good collaterals but no recanalization, only $38 \%$ had good outcomes. Therefore, the presence of good collaterals alone will not prevent unfavorable outcomes and successful recanalization is important [27].

Campbell, et al. evaluated the collateral status and infarction expansion between baseline during the study duration of three to five days using acute magnetic resonance imaging in 88 acute ischemic stroke patients [28]. In 30 patients without reperfusion from day three to five, deterioration in collateral status between baseline and subsequent imaging was strongly associated with infarction volume growth [28].

To date, there is no endovascular randomized clinical trial to address patients with LVO acute ischemic stroke and mild to moderate stroke severity.

Goyal, et al. and HERMES collaborators, in their meta-analysis of the individual patient data from five major mechanical thrombectomy clinical trials, identified a beneficial effect from mechanical thrombectomy for patients across the entire NIHSS [3]. However, only a few patients (177 patients with NIHSS scores $\leqslant 10$ ) with minor strokes were available for analysis. HERMES collaborators suggested that in clinical practice, the treatment of such patients should be determined based on specific clinical and imaging characteristics, knowing the risk of subsequent neurological deterioration in patients with proximal LVO [3].

Bhogal, et al. studied 41 patients with acute ischemic stroke and LVO with low NIHSS scores ( $\leqslant$ 5) [11]. These patients received mechanical thrombectomy along with standard medical therapy. The successful recanalization was achieved in $87.8 \%$ patients (thrombolysis in cerebral infarction [TICI] score $\geqslant 2 b$ ) with only two cases of symptomatic intracranial hemorrhage. At the 90-day follow-up evaluation, 75\% of those studied had a modified Rankin Scale (mRS) score from zero to two. There were only three deaths at the 90-day follow-up [11].

Pfaff, et al. performed a retrospective analysis of 484 stroke patients [29]. Thirty-three patients (6.8\%) with LVO strokes and NIHSS score of eight or below received acute endovascular thrombectomy with successful recanalization. Twenty-six patients (78.7\%) had TICI scores ranging from $2 b$ to three. Only two symptomatic hemorrhage cases were observed. More than 
$90 \%$ of patients had a favorable neurological outcome at 90 days (mRS, 0 to $2: 21$ patients, 63.6\%l mRS 0 to 3: 30 patients, 90.9\%) [29].

Haussen, et al. studied 32 patients with NIHSS scores of $\leqslant 5$ and confirmed LVO via computed tomography angiography. Twenty-two patients (69\%) were assigned to thrombolysis therapy and 10 patients (31\%) were assigned to the endovascular mechanical thrombectomy [4]. Fortyone percent of patients assigned to the medical therapy had worsening symptoms and required urgent acute thrombectomy despite their median NIHSS score of two. The median time of worsening neurological symptoms was approximately 5.2 hours, consistent with Campbell, et al.'s report on the dynamic and exhaustible nature of leptomeningeal collaterals. The successful reperfusion rate (TICI $2 b$ to three) was achieved in all thrombectomy patients. All of the endovascular thrombectomy group patient's 90-day mRS ranged from zero to two, but only $77 \%$ of patients in the thrombolysis grouped had mRS ranging from zero to two. Three patients died in the medical group, but no deaths were noted in the endovascular intervention group. Haussen, et al. demonstrated a shift towards providing endovascular thrombectomy to patients with a lower NIHSS score and LVO stroke presenting with mild symptoms. Nearly $25 \%$ of the medically treated group did not achieve full neurological independence at 90 days $[4,28]$.

Dargazanli, et al. published their study on the impact of acute recanalization therapy on clinical outcomes in patients with low NIHSS scores treated with mechanical thrombectomy [30]. They analyzed 138 patients with acute LVO of the anterior circulation with NIHSS scores < 8 and received acute mechanical thrombectomy. Successful reperfusion (TICI $2 b$ to three) was achieved in $81.2 \%$ of patients. At the 90 -day follow-up, an excellent outcome (mRS zero to one) was achieved in 69 patients $(65.0 \%)$, and favorable outcomes (mRS $\leqslant 2$ ) were achieved in 108 patients (78.3\%). The death occurred in seven patients (5.1\%). These findings are consistent with the previously mentioned studies. The probability of excellent outcomes increased with recanalization grades with a rate of $34.6 \%$ in patients with failed or poor recanalization, $61.7 \%$ in patients with TICI $2 \mathrm{~b}$ reperfusion, and $78.5 \%$ in patients with TICI three reperfusion [30].

This review article has several limitations. The studies mentioned here are retrospective, observational in nature, or self-reported safety outcomes lacking central adjudication of imaging or neurological outcomes, without control group comparators except for Haussen, et al. and his intention-to-treat analysis. The small sample size of the patients treated in these studies does not allow for the recommendation of a minimum NIHSS cutoff for acute mechanical thrombectomy.

Although no randomized clinical controlled trial data are available, cumulative clinical evidence supports treating patients presenting with minor to mild stroke symptoms and LVO with timely endovascular thrombectomy.

\section{Conclusions}

Acute endovascular mechanical thrombectomy in patients presenting with minor to mild stroke symptoms and proximal large vessel occlusion (LVO) appears to be favorable and safe and carries a high chance of successful recanalization with good clinical outcomes. However, future randomized controlled clinical trials are necessary for further evaluation of the benefits of endovascular mechanical thrombectomy compared with the best medical therapy in these patients.

\section{Additional Information}

\section{Disclosures}

Conflicts of interest: In compliance with the ICMJE uniform disclosure form, all authors 
declare the following: Payment/services info: All authors have declared that no financial support was received from any organization for the submitted work. Financial relationships: All authors have declared that they have no financial relationships at present or within the previous three years with any organizations that might have an interest in the submitted work. Other relationships: All authors have declared that there are no other relationships or activities that could appear to have influenced the submitted work.

\section{References}

1. Lima FO, Furie KL, Silva GS, et al: Prognosis of untreated strokes due to anterior circulation proximal intracranial arterial occlusions detected by use of computed tomography angiography. JAMA Neurol. 2014, 71:151-157. 10.1001/jamaneurol.2013.5007

2. Christou I, Burgin WS, Alexandrov AV, et al.: Arterial status after intravenous tPA therapy for ischaemic stroke: a need for further interventions. Int Angiol. 2001, 20:208-13.

3. Goyal M, Menon BK, van Zwam WH, et al: Endovascular thrombectomy after large-vessel ischaemic stroke: a meta-analysis of individual patient data from five randomised trials. Lancet. 2016, 387:1723-1731. 10.1016/S0140-6736(16)00163-X

4. Haussen DC, Bouslama M, Grossberg JA, et al: Too good to intervene? Thrombectomy for large vessel occlusion strokes with minimal symptoms: an intention-to-treat analysis. J Neurointerv Surg. 2016, 10.1136/neurintsurg-2016-012633

5. Campbell BC, Mitchell PJ, Kleinig TJ, et al: Endovascular therapy for ischemic stroke with perfusion imaging selection. N Engl J Med. 2015, 372:1009-1018. 10.1056/NEJMoa1414792

6. Jovin TG, Chamorro A, Cobo E, et al: Thrombectomy within 8 hours after symptom onset in ischemic stroke. N Engl J Med. 2015, 372:2296-2306. 10.1056/NEJMoa1503780

7. Saver JL, Goyal M, Bonafe A, et al: Stent-retriever thrombectomy after intravenous t-PA vs. tPA alone in stroke. N Engl J Med. 2015, 372:2285-2295. 10.1056/NEJMoa1415061

8. Berkhemer OA, Fransen PS, Beumer D, et al: A randomized trial of intraarterial treatment for acute ischemic stroke. N Engl J Med. 2015, 372:11-20. 10.1056/NEJMoa1411587

9. Goyal M, Demchuk AM, Menon BK, et al: Randomized assessment of rapid endovascular treatment of ischemic stroke. N Engl J Med. 2015, 372:1019-1030. 10.1056/NEJMoa1414905

10. Maas MB, Furie KL, Lev MH, et al: National institutes of health stroke scale score is poorly predictive of proximal occlusion in acute cerebral ischemia. Stroke. 2009, 40:2988-2993. 10.1161/STROKEAHA.109.555664

11. Bhogal P, Bucke P, Ganslandt O, et al: Mechanical thrombectomy in patients with M1 occlusion and NIHSS score $\leqslant 5$ : A single-centre experience. Stroke Vasc Neurol. 2016, 5:000052. 10.1136/svn-2016-000052

12. Powers WJ, Derdeyn CP, Biller J, et al.: 2015 American Heart Association/American Stroke Association focused update of the 2013 guidelines for the early management of patients with acute ischemic stroke regarding endovascular treatment. Stroke. 2015, 46:3020-3035. 10.1161/STR.0000000000000074

13. Smith EE, Fonarow GC, Reeves MJ, et al: Outcomes in mild or rapidly improving stroke not treated with intravenous recombinant tissue-type plasminogen activator: findings from get With The Guidelines-Stroke. Stroke. 2011, 42:3110-5.

14. California Acute Stroke Pilot Registry (CASPR) investigators: Prioritizing interventions to improve rates of thrombolysis for ischemic stroke. Neurol. 2005, 64:654-659.

15. Katzan IL, Hammer MD, Hixson ED, et al: Utilization of intravenous tissue plasminogen activator for acute ischemic stroke. Arch Neurol. 2004, 61:346-350.

16. Kleindorfer D, Kissela B, Schneider A, et al: Eligibility for recombinant tissue plasminogen activator in acute ischemic stroke: A population-based study. Stroke. 2004, 35:27-29.

17. Smith EE, Abdullah AR, Petkovska I, et al.: Poor outcomes in patients who do not receive intravenous tissue plasminogen activator because of mild or improving ischemic stroke. Stroke. 2005, 36:2497-2499.

18. Nedeltchev K, Schwegler B, Haefeli T, et al: Outcome of stroke with mild or rapidly improving symptoms. Stroke. 2007, 38:2531-2535.

19. Broderick JP, Palesch YY, Demchuk AM, et al.: Endovascular therapy after intravenous t-PA versus t-PA alone for stroke. N Engl J Med. 2013, 368:893-903. 10.1056/NEJMoa1214300

20. Kim JT, Park MS, Chang J, et al.: Proximal arterial occlusion in acute ischemic stroke with low 
NIHSS scores should not be considered as mild stroke. PLoS One. 2013,

10.1371/journal.pone.0070996

21. Mokin M, Masud MW, Dumont TM, et al: Outcomes in patients with acute ischemic stroke from proximal intracranial vessel occlusion and NIHSS score below 8. J Neurointerv Surg. 2014, 6:413-417. 10.1136/neurintsurg-2013-010720

22. Cronin CA, Hermann LD: Right hemisphere ischemia is more likely to cause falsely "mild" symptoms and poor outcomes without thrombolysis. J Neurol Transl Neurosci. 2014, 2:1036.

23. Shin N-Y, Kim K, Park M, et al: Dual-phase CT collateral score: a predictor of clinical outcome in patients with acute ischemic stroke. PLoS One. 2014,

24. van den Wijngaard IR, Boiten J, Holswilder G, et al: Impact of collateral status evaluated by dynamic computed tomographic angiography on clinical outcome in patients with ischemic stroke. Stroke. 2015, 46:3398-3404. 10.1161/STROKEAHA.115.010354

25. Lima FO, Furie KL, Silva GS, et al: The pattern of leptomeningeal collaterals on CT angiography is a strong predictor of long-term functional outcome in stroke patients with large vessel intracranial occlusion. Stroke. 2010, 41:2316-2322.

10.1161/STROKEAHA.110.592303

26. Berkhemer OA, Jansen IG, Beumer D, et al: Collateral status on baseline computed tomographic angiography and intra-arterial treatment effect in patients with proximal anterior circulation stroke. Stroke. 2016, 47:768-776. 10.1161/STROKEAHA.115.011788

27. Miteff F, Levi CR, Bateman GA, et al: The independent predictive utility of computed tomography angiographic collateral status in acute ischaemic stroke. Brain. 2009, 132:22312238. 10.1093/brain/awp155

28. Campbell BCV, Christensen S, Tress BM, et al.: Failure of collateral blood flow is associated with infarct growth in ischemic stroke. J Cereb Blood Flow Metab. 2013, 33:1168-1172. 10.1038/jcbfm.2013.77

29. Pfaff J, Herweh C, Pham M, et al: Mechanical thrombectomy in patients with acute ischemic stroke and lower NIHSS scores: recanalization rates, periprocedural complications, and clinical outcome. AJNR Am J Neuroradiol. 2016, 37:2066-2071.

30. Dargazanli C, Consoli A, Gory B, et al: Is reperfusion useful in ischaemic stroke patients presenting with a low national institutes of health stroke scale and a proximal large vessel occlusion of the anterior circulation?. Cerebrovasc Disc. 2017, 43:305-312. $10.1159 / 000468995$ 